Meta

Journal des traducteurs

Translators' Journal

\title{
Doyen ou doyenne ?
}

\section{Christine Bagge}

Volume 30, numéro 3, septembre 1985

URI : https://id.erudit.org/iderudit/001946ar

DOI : https://doi.org/10.7202/001946ar

Aller au sommaire du numéro

Éditeur(s)

Les Presses de l'Université de Montréal

ISSN

0026-0452 (imprimé)

1492-1421 (numérique)

Découvrir la revue

Citer cet article

Bagge, C. (1985). Doyen ou doyenne ? Meta, 30(3), 255-256.

https://doi.org/10.7202/001946ar

Ce document est protégé par la loi sur le droit d'auteur. L'utilisation des services d'Érudit (y compris la reproduction) est assujettie à sa politique d'utilisation que vous pouvez consulter en ligne.

https://apropos.erudit.org/fr/usagers/politique-dutilisation/
Cet article est diffusé et préservé par Érudit.

Érudit est un consortium interuniversitaire sans but lucratif composé de l’Université de Montréal, l'Université Laval et l'Université du Québec à Montréal. Il a pour mission la promotion et la valorisation de la recherche. https://www.erudit.org/fr/ 


\section{DOYEN OU DOYENNE ?}

Il s'agit là d'une question d'actualité puisque de plus en plus de doyens de faculté sont des " doyennes".

En fait, pourquoi la question, puisque les dictionnaires unilingues de langue française donnent comme entrée ' doyen,enne'. On rencontre, néanmoins, une certaine réticence de la part des locuteurs à utiliser 'doyenne' lorsqu'il s'agit du " titre de la première dignité dans les facultés d'une université " (acception $\mathrm{n}^{\circ} 2$ dans le Petit Robert). Ceci est probablement dû au fait qu'alors que pour la première acception ("Titre de dignité ecclésiastique") un exemple au féminin ("Doyenne d'une abbaye") complète l'exemple au masculin, tel $n^{\prime}$ 'est pas le cas pour l'acception $n^{\circ} 2$ précitée. Il est cependant assez facile de réfuter cet argument.

Tout d'abord rien dans la notation pratique du dictionnaire en question n'indique qu'il existe une différence fondamentale entre les acceptions 1 et 2 , dont la formulation est quasi identique ("Titre de... »), qui interdirait dans le deuxième sens la forme féminine courante dans le premier sens. Aucune contrainte linguistique n'est présente, mais tout simplement une réalité extra linguistique est reflétée dans le dictionnaire - les doyennes d'abbaye existent depuis longtemps, alors que les doyennes de faculté sont un phénomène récent. En période de transition, il y a toujours un certain décalage entre le moment où le changement se produit et celui où il est enregistré dans le dictionnaire, ce qui ne veut pas dire que, le cas échéant, l'expression ne peut pas être utilisée puisque, idéalement, le dictionnaire n'est là que pour entériner l'usage.

Il est d'ailleurs intéressant de remarquer que le parallélisme qui existe entre les acceptions 1 et 2 se retrouve entre les acceptions 3 et 4 :

3. Personne qui est plus ancien des membres d'un corps, par ordre de réception. 
4. Personne la plus âgée.

Puisque ces définitions commencent toutes deux par "Personne ", il ne fait aucun doute que des deux acceptions peuvent s'appliquer à la fois aux hommes et aux femmes ['Personne' dans le Petit Robert : Être humain (lorsqu'on ne peut ou ne veut préciser [...] le sexe)]. Or, seule la quatrième donne un exemple au féminin.

Il n'est pas question de faire le procès du Petit Robert ou de l'accuser de sexisme, puisque, comme on l'a déjà mentionné, il est probablement le reflet fidèle de la réalité telle qu'elle était à l'époque de sa préparation. Mais, il ne faut pas, non plus, lui faire dire ce qu'il ne dit pas, c'est-à-dire que la forme féminine du mot en question est impossible dans les acceptions 2 et 4.

D'autre part, la Gazette officielle du Québec du 28 juillet 1979 (OLF 1981 : 43) a publié un avis de recommandation qui stipule que " relativement au genre des appellations d'emploi, l'Office de la langue française a recommandé l'utilisation des formes féminines dans tous les cas possibles ". Sont ensuite énumérés quatre procédés de féminisation, avec quelques exemples. 'Doyenne' répond exactement au troisième de ces procédés :

- soit par la création spontanée d'une forme féminine qui respecte la morphologie française. Exemples : députée, chirurgienne, praticienne.

Finalement, l'utilisation de la forme masculine lorsque le référent est une femme peut être considérée comme un anglicisme. La notion de genre joue un rôle négligeable en anglais. Elle n'apparaît généralement que lorsqu'on a à remplacer le nom par un pronom, comme le montre l'exergue suivant :

In order to provide a fair and balanced treatment of both sexes in this publication, the publishers have chosen to alternate the use of masculine and feminine pronouns in preference to the awkwardness encountered by using " he/she ", "his/her ", etc. (Darmon et al. 1981 : vi.)

En anglais, la plupart des mots au sémantème masculin au départ deviennent facilement générique pour s'appliquer également aux femmes (exemple : ' dean'. C'est une évolution qui passe inaperçue parce qu'elle ne requiert pas de processus conscient. Dans les cas rares où un tel processus est nécessaire, l'anglais opte encore pour le terme générique : 'chairman' devient 'chairperson' et non 'charirwoman'. Tel n'est pas le cas en français. Depuis toujours les emplois effectués par des femmes s'expriment au féminin (exemples : tricoteuse, repasseuse, etc.).

Employer 'doyenne' pour donner à une femme le "titre de la première dignité dans les facultés d'une université ", ce n'est pas être féministe à outrance ou négliger la polysémie du mot 'doyen', c'est tout simplement reconnaître une réalité sociale et utiliser le français " avec l'exercice plein et entier de toutes ses possibilités", pour reprendre les mots de Jean Marcel dans le Joual de Troie, en en respectant le génie et en évitant un anglicisme.

\section{Christine BAGGE}

\section{RÉFÉRENCES}

DARMON, R.Y. et al. (1981) : Canadian Marketing, Toronto, McGraw-Hill Ryerson Limited.

OFFICE DE LA LANGUE FRANÇAISE (1981) : Répertoire des avis linguistiques et terminologiques, mai 1979-juillet 1981, Québec, Éditeur officiel du Québec.

ROBERT, P. (1984) : Petit Robert, Paris, les Dictionnaires Robert. 